\title{
UROLOGICAL ONCOLOGY
}

\section{Do patients benefit from routine follow-up to detect recurrences after radical cystectomy and ileal orthotopic bladder substitution?}

Giannarini G, Kessler TM, Thoeny HC, Nguyen DP, Meissner C, Studer UE

Department of Urology University of Berne, Inselspital, Berne, Switzerland

Eur Urol. 2010; 58: 486-94

Background: The need for and intensity of follow-up to detect disease recurrence after radical cystectomy (RC) for transitional cell carcinoma (TCC) remains a matter for debate.

Objective: To determine whether diagnosis of asymptomatic recurrence after RC by routine follow-up investigations confers a survival benefit versus symptomatic recurrence.

Design, Setting, and Participants: Retrospective analysis of 479 patients with nonmetastatic bladder TCC receiving no neoadjuvant chemotherapy/radiation therapy and prospectively followed with a standardised protocol for a median $4.3 \mathrm{yr}$ (range: 0.3-20.9) after RC at an academic tertiary referral centre.

Intervention: RC and extended pelvic lymph node dissection with ileal orthotopic bladder substitution.

Measurements: Cancer-specific survival (CSS) and overall survival (OS) probability for asymptomatic and symptomatic recurrent patients were estimated using the Kaplan-Meier method. The effects of age, nerve-sparing surgery, pathologic tumour stage, lymph node status, adjuvant chemotherapy, mode of recurrence diagnosis, and recurrence site on survival were assessed with multivariable Cox regression models.

Results and Limitations: Of the 174 of 479 patients (36.3\%) with tumour recurrence, 87 were diagnosed by routine follow-up investigations and 87 by symptoms. Routine follow-up mostly detected lung metastases and urethral recurrences, while symptoms were predominantly the result of bone metastases and concomitant pelvic/ distant recurrences. Of 24 patients with urethral recurrences, 13 had carcinoma in situ (CIS). Of these, 12 were successfully managed with urethra-sparing treatment, and 6 are still alive with no evidence of disease. Most other recurrent long-term survivors had lung and extrapelvic lymph node metastases. Cumulative 5-yr survival rates of the entire cohort were $69.8 \%$ (95\% confidence interval [CI], 65.5-74.3\%) for CSS and $61.9 \%(95 \%$ CI, 57.4-66.7\%) for OS. In multivariable analysis, mode of recurrence diagnosis and site of initial recurrence were the only independent predictors of CSS and OS. Patients with recurrences detected by routine follow-up investigations and with secondary urothelial tumours as site of recurrence had a slightly but significantly higher survival probability.

Conclusions: Patients diagnosed with asymptomatic recurrences during our routine follow-up after RC had a slightly higher survival than patients with symptomatic recurrences. Routine follow-up appears particularly effective in early detection of urethral CIS, which can be treated conservatively. In addition, the predominance of lung and extrapelvic lymph node metastases in survivors may justify the use of routine cross-sectional imaging.

\section{Editorial Comment}

Why do patients need follow-up after cystectomy? This retrospective analysis on 174 patients with cystectomy and orthotopic neobladders shows the evidence behind current recommendations of routine follow-up procedures.

Interestingly, only about $7 \%$ of patients had isolated pelvic recurrences whereas about $61 \%$ had distant recurrences only. Ten percent of patients had concomitant local and distant recurrences. Roughly, half of patients were detected without symptoms by routine follow-up procedures. Especially urethral recurrences, which are easily detected by cytology/biopsy, had the potential of cure. 
Thus, routine follow-up including urethral barbotage cytology and routine x-ray analyses are advocated in patients with orthotopic neobladders.

\author{
Dr. Andreas Bohle \\ Professor of Urology \\ HELIOS Agnes Karll Hospital \\ Bad Schwartau, Germany \\ E-mail: boehle@urologie-bad-schwartau.de
}

doi: $10.1590 / S 1677-55382010000700026$

\title{
Multicentric oncologic outcomes of high-intensity focused ultrasound for localized prostate cancer in 803 patients \\ Crouzet S, Rebillard X, Chevallier D, Rischmann P, Pasticier G, Garcia G, Rouviere O, Chapelon JY, Gelet A Department of Urology, Edouard Herriot Hospital, Lyon, France \\ Eur Urol. 2010; 58: 559-66
}

Background: High-intensity focused ultrasound (HIFU) is an emerging treatment for select patients with localized prostate cancer $(\mathrm{PCa})$.

Objectives: To report the oncologic outcome of HIFU as a primary care option for localized prostate cancer from a multicenter database.

Design, Setting, and Participants: Patients with localized PCa treated with curative intent and presenting at least a 2-yr follow-up from February 1993 were considered in this study. Previously irradiated patients were excluded from this analysis. In case of any residual or recurrent $\mathrm{PCa}$, patients were systematically offered a second session. Kaplan-Meier analysis was performed to determine disease-free survival rates (DFSR).

Measurements: Prostate-specific antigen (PSA), clinical stage, and pathologic results were measured pre- and post-HIFU.

Results and Limitations: A total of 803 patients from six urologic departments met the inclusion criteria. Stratification according to d'Amico's risk group was low, intermediate, and high in $40.2 \%, 46.3 \%$, and $13.5 \%$ of patients, respectively. Mean follow-up was 42+/-33 mo. Mean PSA nadir was $1.0+/-2.8 \mathrm{ng} / \mathrm{ml}$ with $54.3 \%$ reaching a nadir of $<$ or $=0.3 \mathrm{ng} / \mathrm{ml}$. Control biopsies were negative in $85 \%$ of cases. The overall and cancerspecific survival rates at 8 yr were $89 \%$ and $99 \%$, respectively. The metastasis-free survival rate at 8 yr was $97 \%$. Initial PSA value and Gleason score value significantly influence the DFSR. The 5- and 7-yr biochemical-free survival rates (Phoenix criteria) were $83-75 \%, 72-63 \%$, and $68-62 \%(\mathrm{p}=0.03)$ and the additional treatment-free survival rates were $84-79 \%, 68-61 \%$, and $52-54 \%$ ( $\mathrm{p}<0.001)$ for low-, intermediate-, and high-risk patients, respectively. PSA nadir was a major predictive factor for HIFU success: negative biopsies, stable PSA, and no additional therapy.

Conclusions: Local control and DFSR achieved with HIFU were similar to those expected with conformal external-beam radiation therapy (EBRT). The excellent cancer-specific survival rate is also explained by the possibility to repeat HIFU and use salvage EBRT.

\section{Editorial Comment}

High-intensity focused ultrasound (HIFU) is not regarded an established treatment in prostate cancer patients as radical prostatectomy and radiation therapy are. Therefore, reports on the long-term outcomes of 